\title{
Spatiotemporal evolution of radio wave pump-induced ionospheric phenomena near the fourth electron gyroharmonic
}

\author{
M. Ashrafi, ${ }^{1,4}$ M. J. Kosch, ${ }^{1}$ K. Kaila, ${ }^{2}$ and B. Isham ${ }^{3}$ \\ Received 27 June 2006; revised 15 January 2007; accepted 20 February 2007; published 30 May 2007.
}

[1] On 12 November 2001, the European Incoherent Scatter (EISCAT) high-frequency (HF) radio wave transmitter facility, operating in O-mode at $5.423 \mathrm{MHz}$ with $550 \mathrm{MW}$ effective radiated power, produced artificial optical rings which appeared immediately at transmitter turn-on and collapsed into blobs after $\sim 60 \mathrm{~s}$ while descending in altitude. A similar descent in altitude was observed in the EISCAT ultra high frequency (UHF) ion line enhancements. Likewise, the stimulated electromagnetic emission (SEE) spectra changed as the pump frequency approached the fourth electron gyroharmonic due to pump-induced variations in electron concentration. Optical recordings were made from Skibotn at 630.0 and $557.7 \mathrm{~nm}$ and from Ramfjord in white light. The altitude of the initial optical ring and steady state blob has been estimated by triangulation. The evolution in altitude of the optical emissions, ion line enhancements, and SEE spectra all show a similar morphology but are generally not at exactly the same height. Typically, the optical height is close to and a few kilometers below that of the radar backscatter but sometimes above it, both of which are above the SEE generation altitude. There is evidence that upper hybrid (UH) waves, which propagate perpendicular to the magnetic field line, and Langmuir (L) waves, which propagate parallel to the magnetic field line, act simultaneously to accelerate electrons even in the steady state.

Citation: Ashrafi, M., M. J. Kosch, K. Kaila, and B. Isham (2007), Spatiotemporal evolution of radio wave pump-induced ionospheric phenomena near the fourth electron gyroharmonic, J. Geophys. Res., 112, A05314, doi:10.1029/2006JA011938.

\section{Introduction}

[2] High-frequency (HF) O-mode electromagnetic (EM) pump waves may excite a number of instabilities in the ionospheric plasma. On the timescale of milliseconds, the parametric decay instability (PDI) near the reflection layer of the pump wave leads to the enhancement of the electrostatic Langmuir (L) waves traveling parallel to the magnetic field line [DuBois et al., 1993]. Electron Langmuir (L) and ion acoustic (IA) waves near the pump reflection altitude may be produced by either weakly turbulent parametric decay instabilities $\left(\mathrm{PDI}_{\mathrm{L}}\right)$ or the strongly turbulent oscillating two-stream instability $\left(\mathrm{OTSI}_{\mathrm{L}}\right.$ ) [e.g., Fejer, 1979; DuBois et al., 1993]. The Langmuir turbulence occurs just below the reflection height of the pump wave and causes enhancements in incoherent backscatter power which is known as the ion line enhancement effect [Jones et al., 1986; Birkmayer et al., 1986; Isham et al., 1999a; Rietveld et al.,

\footnotetext{
${ }^{1}$ Department of Communications Systems, Lancaster University, Lancaster, UK.

${ }^{2}$ Department of Physical Sciences, University of Oulu, Oulu, Finland.

${ }^{3}$ Department of Electrical Engineering, Interamerican University of Puerto Rico, Bayamón, Puerto Rico, USA.

${ }^{4}$ Now at School of Physics and Astronomy, University of Southampton, Southampton, UK.
}

Copyright 2007 by the American Geophysical Union. 0148-0227/07/2006JA011938\$09.00
2000; Djuth et al., 2004]. These enhancements are detected by incoherent scatter radars at the matching height, where the pump frequency $\left(f_{0}\right)$ matches the Langmuir waves' frequency $\left(f_{\mathrm{L}}\right)$ with their wave vectors $(k)$ equal to twice of that of the probing ultra high frequency (UHF) radar wave $\left(k_{\mathrm{UHF}}\right)$, i.e., $f_{0}=f_{\mathrm{L}}\left(k=2 k_{\mathrm{UHF}}\right)$ [Rietveld et al., 2000]. The HF reflection and matching altitudes can be up to $10 \mathrm{~km}$ apart and are simply related [Stubbe et al., 1992].

[3] In addition, the thermal parametric instability (TPI) near the upper hybrid (UH) resonance altitude, which occurs a few kilometers below the pump wave reflection altitude, stimulates UH waves propagating across the magnetic field lines. Small-scale $(\sim 10 \mathrm{~m})$ field-aligned striations (FAS), or density depletions, are the result of UH turbulence, which can be excited by the thermal oscillating two-stream instability [e.g., Das and Fejer, 1979; Dysthe et al., 1983]. Large-scale FAS (hundreds of meters to kilometers) are the result of thermal self-focusing instabilities [e.g., Grach et al., 1977; Guzdar et al., 1996]. UH waves trapped inside the FAS cause ohmic heating of the plasma inside the depleted region which results in further depletion and growth of the field-aligned irregularities over a timescale of $\sim 100 \mathrm{~ms}$ to few seconds [e.g., Vas'kov and Gurevich, 1976; Dysthe et al., 1982]. This effect leads to wide-band attenuation of HF probing radar waves passing through the irregularities in the vicinity of the UH resonance altitude due to scattering, a process known as anomalous absorption [Stubbe et al., 1982; Jones et al., 1984; 
Robinson, 1989]. Besides TPI, UH or electron Bernstein (EB) waves (electron electrostatic waves perpendicular to the field lines) coupled to lower hybrid (LH) waves may also be produced by parametric decay of the pump wave, i.e., $\mathrm{PDI}_{\mathrm{UH} / \mathrm{EB}}: \mathrm{EM} \rightarrow \mathrm{UH} / \mathrm{EB}+\mathrm{LH}$ [Istomin and Leyser, 1995].

[4] Langmuir and UH turbulence are both known to generate radio waves which can be detected on the ground as stimulated electromagnetic emissions (SEE). A number of processes have been suggested in the creation of these emissions, including scattering of the enhanced Langmuir or UH waves from natural or artificial plasma inhomogeneities, for example, FAS, or damped ion acoustic waves and subsequent conversion to EM waves [Thidé et al., 1983; Leyser, 2001].

[5] Electrons accelerated by HF pump-driven electrostatic turbulence can travel up and down the magnetic field lines and excite the neutrals by collisions, stimulating optical emissions identical to the natural aurorae. The dominant red and green line emissions at 630.0 and $557.7 \mathrm{~nm}$ from the two lowest excited states of atomic oxygen, $\mathrm{O}\left({ }^{1} \mathrm{D}\right)$ and $\mathrm{O}\left({ }^{1} \mathrm{~S}\right)$, can be stimulated by electrons with excitation energies more than 1.96 and $4.17 \mathrm{eV}$, respectively [Haslett and Megill, 1974; Bernhardt et al., 1991]. Intensities up to $\sim 200$ Rayleighs $(\mathrm{R})$ for red line and $\sim 50 \mathrm{R}$ for green line emission have been observed in high-latitude HF pumping experiments [Pedersen et al., 2003]. It has been long known that thermal electrons are responsible for some of the $\mathrm{O}\left({ }^{1} \mathrm{D}\right)$ emissions observed in past ionospheric pumping experiments [Haslett and Megill, 1974; Mantas, 1994; Mantas and Carlson, 1996]. However, the red and green line emission intensities provide direct evidence that the thermally heated electrons do not have sufficient fluxes to produce the observed intensities [Gustavsson et al., 2002; Kosch et al., 2004; Ashrafi et al., 2006]. Therefore electrons accelerated by pump-driven electrostatic turbulence are a primary contributor to the excitation of the red line emission. The green line because of its higher threshold energy can only be due to HF pump-driven accelerated electrons [Gustavsson et al., 2002]. However, the electron acceleration mechanism is still a subject of debate.

[6] Electron acceleration by UH turbulence at high latitudes was first suggested by Leyser et al. [2000]. This hypothesis has been supported by other theoretical work [Istomin and Leyser, 2003] and experimental evidence [Kosch et al., 2002; Gustavsson et al., 2004] provided by means of pumping close to electron gyroharmonic frequencies. In a more recent study by Grach et al. [2004] at the Sura ionospheric heating facility in Russia, the UH or EB waves are shown to be responsible for $557.7 \mathrm{~nm}$ airglow enhancements. However, based on other theoretical considerations and previous experiments, it has been shown that Langmuir turbulence is also responsible for the accelerated electrons causing artificial optical emissions [Gurevich et al., 1985; Bernhardt et al., 1989; Kosch et al., 2004]. Gumerov et al. [1999] provided evidence of pump-induced $557.7 \mathrm{~nm}$ optical emissions in the first $5 \mathrm{~ms}$ of the pump-on cycle, which is much shorter than the growth time of the FAS $(\sim 1-10 \mathrm{~s})$, which is clear evidence of electron acceleration by Langmuir turbulence due to parametric instabilities.

[7] The coexistence of the thermal (TPI) and parametric $\left(\mathrm{PDI}_{\mathrm{UH} / \mathrm{EB}}\right)$ instabilities has been suggested by [Mishin et al., 2005b], in which both UH and Langmuir turbulence are thought to contribute to the production of the airglow. It has been established that the generation mechanism for Langmuir turbulence at angles close to the magnetic zenith is different from the $\mathrm{PDI}_{\mathrm{L}}$ process at the reflection altitude of the pump wave near the local geographic zenith [Mishin et al., 2005a]. By pumping at angles inside the Spitze cone (approximately $6^{\circ}$ zenith angle at the European Incoherent Scatter (EISCAT)), the pump waves reach the standard reflection altitude where the local plasma frequency equals the pump frequency [Rietveld et al., 1993]. The Spitze angle is equal to $\arcsin \left(\left[(Y /(1+Y)]^{1 / 2} \sin \theta_{\mathrm{B}}\right)\right.$, where $\theta_{\mathrm{B}}$ is the angle of the geomagnetic field from vertical and $Y=f_{\text {ce }} / f_{0}$, where $f_{\text {ce }}$ is the electron cyclotron frequency and $f_{0}$ is the pump frequency. O-mode waves with incident angles outside the Spitze angle, for example, at magnetic zenith, reflect below the standard reflection layer. Mishin et al. [2004] developed a new scenario for the generation of Langmuir waves outside the Spitze cone through the oscillating two-stream instability of UH waves in the magnetic zenith direction [Kuo et al., 1997]. An additional mechanism has been suggested in which electrons can also be accelerated by LH turbulence and produce optical emissions [Mishin et al., 2004].

[8] In this paper, we present results from an ionospheric O-mode pumping experiment near the 4th electron gyroharmonic at the EISCAT heating facility. This unique experiment provided the first observational evidence that accelerated electrons through Langmuir turbulence produce the artificial optical emissions at high latitudes [Kosch et al., 2004]. A unique feature is that only by pumping $9^{\circ}$ south of local geographic zenith did an optical annular structure appear, collapsing into a blob in less than $60 \mathrm{~s}$. Many aspects of this experiment remain unexplained; for example, why the optical emissions form around the pump beam edge where the HF power is more than half reduced or why the annular structure only occurs for one specific pump beam zenith angle ( $9^{\circ}$ south). In the following sections, we describe and analyze the spatial and temporal evolution of the UHF radar backscatter, the optical emission height using triangulation, and the SEE spectra including a deduction of their generation altitude based on their spectral shape.

\section{Stimulated Electromagnetic Emissions: The Broad Up-Shifted Maximum (UM)}

[9] The first observations of stimulated electromagnetic emissions (SEE) were reported in Tromsø by Thidé et al. [1982] during ionospheric pump experiments. The stimulated emissions spectra, which cover about $-100 \mathrm{kHz}$ to $+200 \mathrm{kHz}$ of bandwidth around the reflected HF pump wave [Frolov et al., 1998], with intensities up to a few tens of decibels above the background noise level, can be detected on the ground. Using the SEE spectra, it is possible to study the ionospheric processes directly and without using additional EM probing waves. Scattering of the excited plasma waves off small-scale irregularities near the UH resonance altitude has been long considered as the main generation mechanism for the SEE [Grach, 1985]. There have been attempts to link different SEE spectral properties, and their temporal evolution, to the development of various wave interaction processes in the artificially perturbed ionosphere [Leyser, 2001 and references therein]. It is known that the SEE features and their timescales are associated with the 
ponderomotive force which acts during the first $\sim 10 \mathrm{~ms}$ and ponderomotive/thermal type processes which develop in timescales of $\sim 1$ s of pumping [Leyser, 2001].

[10] Experimental observations have shown that most of the SEE spectral characteristics depend on the pump frequency $\left(f_{0}\right)$ in relation to the F-region electron cyclotron frequency $\left(f_{\text {ce }}\right)$ [Leyser et al., 1989, 1990; Stubbe et al., 1994; Honary et al., 1999; Cheung et al., 1998] and/or F-region critical frequency [Leyser et al., 1990; Armstrong et al., 1990]. The first classification of the SEE spectral features was performed by Stubbe et al. [1984] who categorized them either as "gyrofeatures" which exist only at $f_{0} \approx n f_{\text {ce, }}$, where electron gyroharmonic number $n=3$ or greater, such as the up-shifted maximum (UM), or universal features, which exist at all frequencies except for pumping close to a gyroharmonic, for example, the down-shifted maximum (DM).

[11] The most prominent gyrofeature of the up-shifted SEE spectra is the broad up-shifted maximum (BUM), which is strongly dependent on the pump frequency. The BUM is known to occur at pump frequencies close to and slightly above the electron gyroharmonics, and it normally appears in the SEE spectra just above the pump frequency [Leyser et al., 1990; Stubbe et al., 1994]. The BUM peak frequency obeys the following empirical relation, $f_{\mathrm{BUM}}=$ $2 f_{0}-n f_{\text {ce }}$, in which $f_{\mathrm{BUM}}$ is the frequency of the BUM peak intensity [Leyser et al., 1989]. When pumping near an electron gyroharmonic, due to the strong cyclotron damping of the waves traveling perpendicular to the magnetic field line, the BUM and DM structures cease to exist. For $f_{0}>n f_{\text {ce, }}$, EB and UH waves can be enhanced and lead to the generation of the BUM features for frequencies near the electron gyrofrequency [Leyser et al., 1989].

[12] Previous experiments have demonstrated that anomalous absorption of HF probing waves, and therefore FAS, is suppressed near the electron gyroharmonics [Robinson, 1989]. Some SEE features such as the down-shifted maximum (DM) and broad continuum (BC) are also suppressed at the same range of frequencies [Stubbe et al., 1994]. In addition, the BUM risetime is similar to some of the other features of the SEE, such as the DM, and is consistent with the growth time of the FAS [Leyser et al., 1990; Frolov et al., 1996]. Therefore FAS play a major role in the generation of such emissions. The DM feature of the SEE suppresses only in a narrow range of frequencies in the vicinity of the electron cyclotron frequency where the FAS are suppressed. However, features such as the BUM demonstrate a rather more complex behavior [Frolov et al., 1996]. The BUM starts to appear at pump frequencies $\sim 10 \mathrm{kHz}$ below the gyroharmonic frequency (for $n=5$ ) [Frolov et al., 1996], it maximizes around $30-40 \mathrm{kHz}$ above the electron gyroharmonic [Leyser, 2001], and continues to exist to a maximum pump frequency of $\sim 100 \mathrm{kHz}$ above the gyrofrequency [Thidé et al., 1983; Stubbe et al., 1994]. During this frequency interval, the FAS are suppressed only in a very narrow range of frequencies, and despite further development of the FAS for frequencies above this range, the BUM structure ceases to exist for higher pump frequencies. Therefore the importance of the FAS in the generation mechanism of the BUM emission is not entirely clear [Wagner et al., 1999].

[13] There have been attempts to provide theoretical models to describe the properties of the BUM spectra in the past [Leyser, 2001, and references therein]. However, none of the theoretical explanations has been able to describe all of the observational properties of this phenomenon [Frolov et al., 1998]. Various four-wave generation mechanisms have been frequently suggested in the literature due to the up-shifted characteristic of the BUM. These explanations mostly involve EB and $\mathrm{UH}$ waves both of which are generated by thermal instabilities near the UH resonance altitude.

\section{Radar Backscatter}

[14] Artificial field-aligned irregularities are a common feature of $\mathrm{HF} \mathrm{O}$-mode pumping, resulting from mode conversion of the EM pump waves into electrostatic waves at the UH resonance altitude [Robinson, 1989], a few kilometers below the HF reflection altitude. Bragg scattering of the HF sounding waves from preexisting natural or artificially stimulated irregularities with a scale size of half the radar wavelength produces the HF coherent backscatter that is used as a proxy for detecting the excited UH waves [Stubbe, 1996].

[15] By pumping the ionosphere close to the third or higher electron gyroharmonic frequencies, the growth of UH waves and FAS is suppressed. This allows most of the pump energy to reach the HF reflection height where Langmuir turbulence-related processes dominate the electron heating [Honary et al., 1999]. This phenomenon is commonly observed as persistent ion line enhancements in the incoherent scatter radar spectra occurring just below the pump wave reflection altitude. It has been attributed to Langmuir processes in which the pump wave, as a result of parametric instabilities, couples to a high-frequency plasma wave and a low frequency IA wave [Bernhardt et al., 1989; Stubbe et al., 1992; Djuth et al., 1994; Kosch et al., 2004]. The persistent enhancements are only visible at zenith angles close to the direction of the magnetic field line [Isham et al., 1999b; Kosch et al., 2004, Figure 3].

[16] Since the threshold for the parametric decay instability is easily exceeded, the enhanced Langmuir waves can become pump waves themselves and excite secondary parametric instabilities [Rietveld et al., 1993]. As a result, a Langmuir wave can further decay into an IA wave and another Langmuir wave with a lower frequency. This cascading process continues as long as the threshold for excitation of the instability is met. Langmuir waves cascade in frequency and broaden the plasma and ion line radar frequency spectrum [Kohl et al., 1993]. The cascade lines of the up- and down-propagating plasma waves can only be excited at different altitudes where their frequency matches the local plasma mode. The IA waves connected to cascade processes are detected at the altitudes where ion waves produce nonlinear Landau damping cascades. These echoes contribute to the backscatter ion line enhancements in the vicinity of the O-mode reflection altitude [Djuth et al., 1994]. A radar with sufficient range resolution should detect (1) the primary ion and plasma line and (2) the cascade ion and plasma lines. Nevertheless, if the resolution of the radar is comparable to the separation of the excitation altitudes, only one ion line enhancement will be observable [Stubbe et al., 1992]. This is the case for the data analyzed here. The EISCAT UHF radar data have a range resolution of $\sim 5.4 \mathrm{~km}$ which is similar to the estimated difference in height between 
the primary and cascade ion lines using equations (11) and (12) in the work by Stubbe et al. [1992] ( 3 km). Therefore the radar range resolution is not sufficient to resolve the two different signatures.

\section{Experiment Description}

[17] On 12 November 2001, the EISCAT heating facility [Stubbe, 1996] was transmitting O-mode waves at $5.423 \mathrm{MHz}$ operating at $550 \mathrm{MW}$ effective radiative power (ERP) with a 2-min on, 2-min off cycle with the pump beam pointed $9^{\circ}$ south of vertical in the first half of the experiment $(15: 08-$ 16:55 UT). During the second half (16:57-20:00 UT), the pump beam was scanning between $3^{\circ}$ north and $15^{\circ}$ south of vertical in $3^{\circ}$ steps with a 2 -min on, 1-min off pump cycle. The pump beam width is $\sim 7^{\circ}$ throughout and magnetic zenith is $12.8^{\circ}$ south of vertical. The pump frequency corresponds to the fourth electron gyroharmonic at $215 \mathrm{~km}$, which is close to the HF interaction altitude during the experiment.

[18] Optical observations were made simultaneously from two stations $\sim 52 \mathrm{~km}$ apart. The Digital All-Sky Imager (DASI) camera [Kosch et al., 1998] with a $50^{\circ}$ field of view was located remotely at Skibotn, Norway $\left(69.35^{\circ} \mathrm{N}\right.$ and $20.36^{\circ} \mathrm{E}$ ), pointing vertically using narrow-band interference filters at $630.0 \mathrm{~nm}$ for 15:08-16:21 UT as well as 18:1320:25 UT and 557.7 nm for 16:24-18:09 UT. The other camera situated at Ramfjordmoen $\left(69.59^{\circ} \mathrm{N}\right.$ and $\left.19.23^{\circ} \mathrm{E}\right)$ colocated with EISCAT and was looking along the magnetic field line direction with a $\sim 40^{\circ}$ field of view, recording in white light. The DASI used an integration time of $5 \mathrm{~s}$ for $557.7 \mathrm{~nm}$ and $10 \mathrm{~s}$ for $630.0 \mathrm{~nm}$, whereas the Ramfjordmoen camera used a 13-s cycle with a 3-s integration.

[19] Simultaneous ion line observations using the EISCAT UHF radar [Rishbeth and van Eyken, 1993] were performed with the radar beam sweeping in $3^{\circ}$ steps, moving from $3^{\circ} \mathrm{S}$ to $15^{\circ} \mathrm{S}$ of the local geographic zenith in a north-south meridian scan from 15:08 to 16:55 UT. Between 16:57 and 20:00 UT, the UHF radar was pointing $13^{\circ} \mathrm{S}$ of vertical. The radar signature of the optical ring and its descent in altitude were observed in the EISCAT UHF backscatter power enhancements near the reflection altitude [Kosch et al., 2004]. Similar pump-induced descending radar signatures (not rings) have been observed by Djuth et al. [1994] as well as Dhillon and Robinson [2005]. For details of the optical and radar observations, refer to the work of Kosch et al. [2004].

[20] The Super Dual Auroral Radar Network (SuperDARN) Co-operative UK Twin Auroral Sounding System (CUTLASS) Hankasalmi coherent scatter radar was operating for an unrelated experiment. Although data are available for the period of our experiment, the time and range resolution over EISCAT is poor (range resolution of $45 \mathrm{~km}$, integration time of $7 \mathrm{~s}$ with a 2-min beam scan time), giving a single measurement $70 \mathrm{~s}$ after pump-on only.

[21] SEE recordings were also made for the entire period of the experiment. The data recordings have been performed with a 14-s integration time and $300-\mathrm{Hz}$ frequency resolution covering -100 to $+200 \mathrm{kHz}$ around the pump frequency.

\section{Observations and Data Analysis}

[22] The optical signature produced by the pump was a ring-shaped structure at the start of the pump-on period which collapsed into a blob after $\sim 60 \mathrm{~s}$ of pumping [Kosch et al., 2004]. Figure 1 shows the $10 \mathrm{~s}$ integrated optical emissions recorded by the DASI at $557.7 \mathrm{~nm}$ for the Heater cycle starting at 16:25 UT (top panel) and 17:24 UT (bottom panel). Artificial optical annuli, which were centered on the pump beam, formed a cylinder of optical emission, parallel to the magnetic field line direction. Intensities up to $\sim 100 \mathrm{R}(50 \mathrm{R})$ for the ring and $\sim 300 \mathrm{R}(100 \mathrm{R})$ for the blob structure artificial aurora were observed in $630.0 \mathrm{~nm}$ (557.7 nm).

[23] The optical artificial aurora forms a ring structure just outside the pump wave $-3 \mathrm{~dB}$ contour and collapses into a blob along the magnetic field line. Investigating the structure of the optical emissions reveals that, at the start of each pumping cycle, the optical signature may take a variety of forms throughout the experiment. In the first part of the experiment, the annuli form an obvious ring which is mostly brighter at angles closer to the vertical and inside the Spitze angle (Figure 1, top panel) before collapsing into a blob in the magnetic zenith. Later on in the experiment (after 17:24 UT), the optical emission forms a blob in the magnetic zenith with a bright crescent outside the Spitze cone, which collapses into a blob only in the magnetic zenith (Figure 1, bottom panel). The reason for the formation of the annuli structure, which appears where the gradient of the pump waves' intensity is the highest, is not known. From 17:45 UT onwards, when the reflection height increases abruptly, the optical structure forms only as a blob and not as a ring. There are few cases after 17:45 UT in which the optical emissions are present despite the pump frequency being above $f_{0} F 2$. Pedersen et al. [2003] and Kosch et al. [2005] have provided experimental evidence that the brightest optical emissions occur for pump frequencies close to the F-layer critical frequency with an emission cutoff at about $0.5 \mathrm{MHz}$ above $f_{0} F 2$.

[24] Multistation observations of auroral emissions and the application of height triangulation in estimating the location of the emission are well-known, long-standing techniques [Störmer, 1955]. In the case of artificial ionospheric modification, altitude estimation of the pump-induced optical emissions has been performed in the past using tomography [Gustavsson et al., 2001], height triangulation [Haslett and Megill, 1974], and parallax [Kosch et al., 2004].

[25] On 12 November 2001, the optical recordings of the artificial optical emissions were performed from two stations located at Skibotn and Ramfjordmoen with about $52 \mathrm{~km}$ separation. The green and red line data from the DASI camera together with the white light recordings of the Ramfjordmoen camera have been used to estimate the height of the emissions. We assume that the vertical extent of the artificial aurora is limited, or equivalently, the height triangulation focuses on the region of greatest intensity, i.e., near the bottom of the forms due to increasing $\mathrm{O}$ density with decreasing altitude. The $\mathrm{O}\left({ }^{1} \mathrm{~S}\right) 557.7 \mathrm{~nm}$ emission is suitable for studying temporal variations, due to its short radiative lifetime $(\sim 0.7 \mathrm{~s})$, compared with the brighter $\mathrm{O}\left({ }^{1} \mathrm{D}\right) 630.0 \mathrm{~nm}$ emission with $\sim 30$ s effective lifetime in the F-layer [Gustavsson et al., 2001]. Therefore for the first half of the experiment (until 16:21 UT) when the Skibotn camera was recording the red line emission, only the height of the optical emission just before the pump-off period has been calculated, when the steady state is most likely. For the 

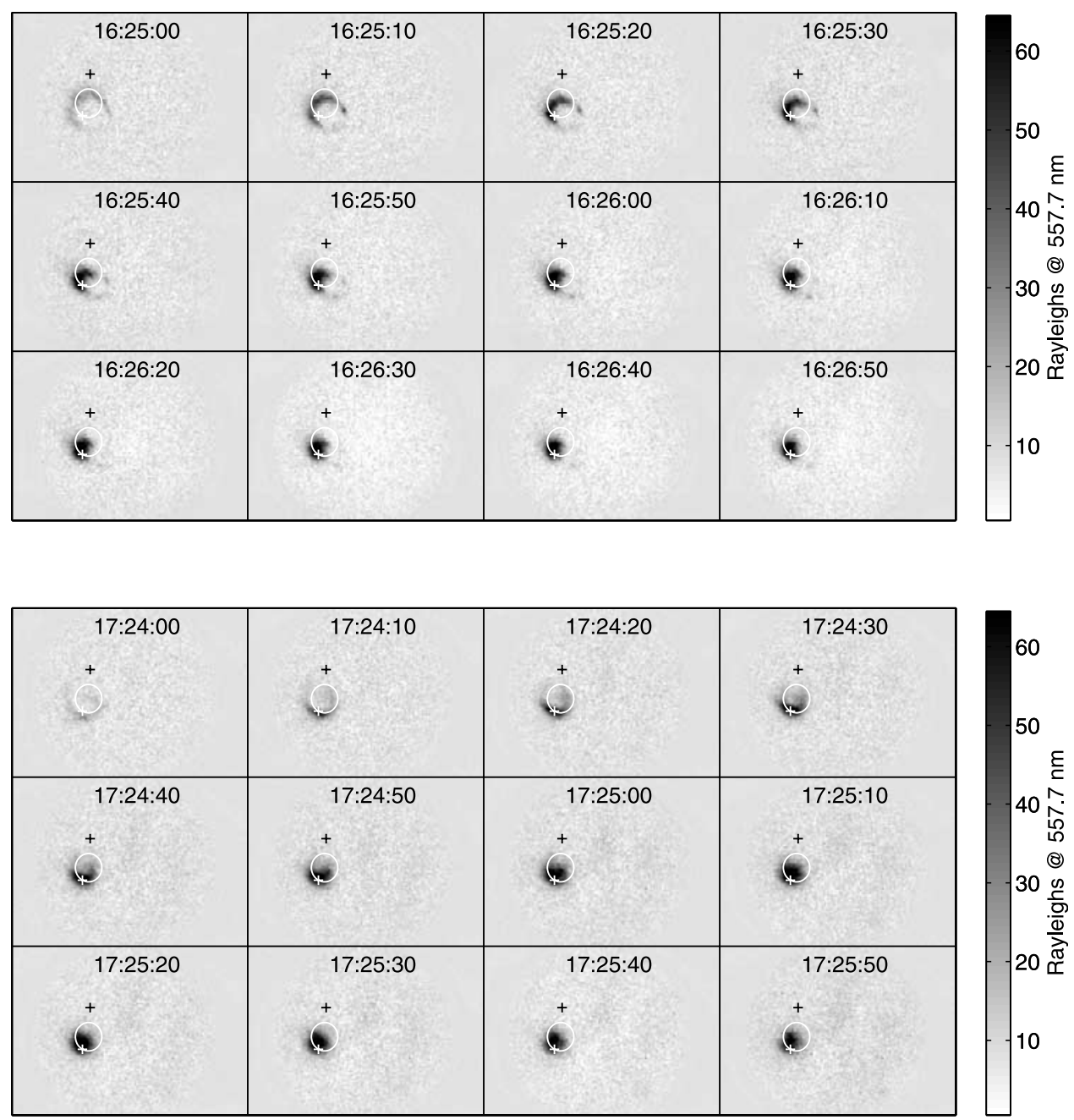

Figure 1. Series of the DASI camera images showing the development of the pump-induced optical emissions at $557.7 \mathrm{~nm}$ with a 5-s integration after background subtraction and filtering. The circles mark the $-3 \mathrm{~dB}$ pump beam projected at $230 \mathrm{~km}$ altitude and tilted $9^{\circ}$ south of the local geographic zenith at Ramfjordmoen. The upper cross indicates the location of the HF transmitter and the lower cross shows the magnetic field line direction at Ramfjordmoen projected at $230 \mathrm{~km}$ altitude. Top panel, the Heater turns on at 16:25 UT and turns off at 16:27 UT. Bottom panel, the Heater turns on at 17:24 UT and turns off at 17:26 UT.

second half of the experiment, when the Skibotn camera recorded with the $557.7 \mathrm{~nm}$ filter, height triangulation was performed every $20 \mathrm{~s}$, synchronized to the pump-on cycle.

[26] In order to correct the lens distortion for both cameras and calculate each camera's exact position and pointing direction, star field images, computed from a star catalog and mapped onto the data images, have been used [Duffet-Smith, 1990]. Second-order corrections have been made to the image $x$ and $y$ directions in order to compensate for the lens distortion-induced discrepancies between the recorded images and the star map, which is typical of night vision imaging systems. The same corrections have to be made to all the images from both cameras [Ashrafi et al., 2005].

[27] In order to eliminate the effect of the unwanted background signal, which also includes numerous stars in the case of the Ramfjordmoen camera recording in white light, background subtraction and filtering have been applied.
A clear picture of only the artificial emissions is obtained by subtracting the average of five successive images of the preceding pump-off cycles from any image during the pump-on cycle of interest. In addition, a $5 \times 5$ median filter has been applied to the images to eliminate the background noise and residual stars due to their motion. The Skibotn and Ramfjord images have been synchronized because the integration and cycle time of the cameras are different. The difference in time between the real camera image and the required synchronized image is only a few seconds; therefore linear interpolation is a good approximation for producing the desired image. This method has been applied to the Ramfjord camera images in order to synchronize them with the DASI data.

[28] For the height triangulation, the synchronized images are transformed into a common coordinate system. Geographic coordinates are well suited to this purpose. First, the image is converted into altitude and azimuth angles in 


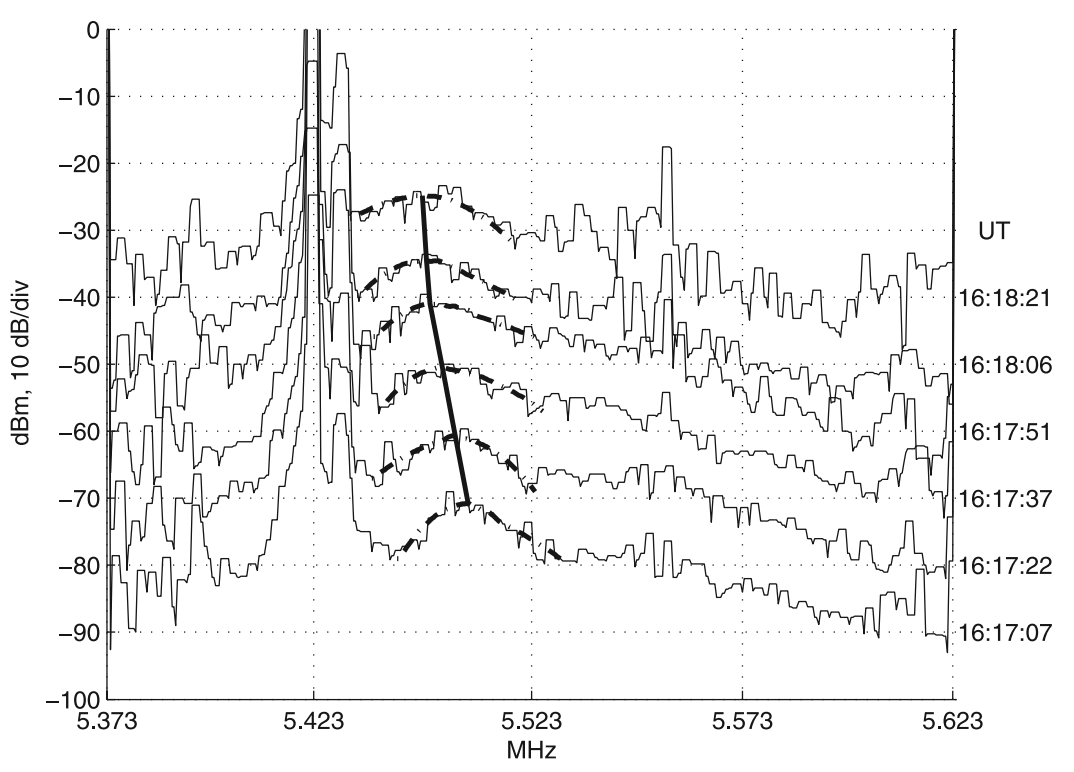

Figure 2. The SEE spectra for the Heater cycle starting at 16:17 UT recorded with a 14-s integration. A $10-\mathrm{dB}$ offset has been added to each sequential spectrum. The broken lines mark the filtered BUM structure which has been used to estimate the peak of the BUM spectrum. The heavy solid line indicates the frequency of the BUM peak. The corresponding time related to each spectrum is indicated in UT.

horizon coordinates [Duffet-Smith, 1990]. Then, by choosing a certain height, the image is transformed into geographic coordinates. Finally, the linear two-dimensional correlation coefficient between the corresponding images in the geographic coordinates from the different platforms has been calculated within a common geographic area between $17.3^{\circ}$ and $20.2^{\circ} \mathrm{E}$ longitude and $68.6^{\circ}$ and $69.6^{\circ} \mathrm{N}$ latitude. The correlation coefficient has been calculated for a range of heights in the vicinity of the pump wave reflection altitude inferred from ionosonde data. The height where the crosscorrelation maximizes is the most likely altitude of the peak optical volume emission rate. This technique has the advantage that no common edge or boundary needs to be identified, and the entire diffuse structure contributes to the height triangulation result, greatly reducing the uncertainty.

[29] The correlation coefficient versus height has been calculated for each 2-km altitude step. By fitting a curve to the altitude versus cross-correlation points, the maximum cross-correlation achieved for any given time has been determined [Ashrafi et al., 2005]. This gives the true equivalent height of the origin of the optical emissions, assuming zero vertical extent. In the ideal case, the cross-correlation for this altitude should be close to unity but considering the measurement uncertainty, lens distortion corrections, time synchronization, noise, and other factors involved (such as vertical extent), this is not generally achievable. Another source of altitude triangulation uncertainty derives from one camera being monochromatic while the other was panchromatic. The typical range of values for the maximum crosscorrelation is $\sim 0.6-0.8$. The error associated with the height estimate is generated by uncertainties in the cross-correlation and is expressed as the standard deviation of the correlation coefficient for each selected altitude. The height estimate uncertainty is $\sim 4-5 \mathrm{~km}$ on average. For details of the height triangulation technique refer to the work by Ashrafi et al. [2005], http://www.des.lancs.ac.uk/iono/publications/
31 am_proceedings.pdf. The results are shown and discussed below.

[30] Using the apparent increased horizontal extension of the optical signature in the DASI images compared to the Ramfjordmoen images, it is possible to estimate the vertical extent of the emission column by mapping the magnetic field line onto the images in geographic coordinates because the Ramfjordmoen camera is colocated with the EISCAT Heater whereas the DASI is $52 \mathrm{~km}$ away. Therefore these latter images have parallax; for example, at 16:25:10 and 18:03:20 UT, the field-aligned extension of the $557.7 \mathrm{~nm}$ artificial aurora to first order is about 70 and $100 \mathrm{~km}$, respectively, which is consistent with that by Kosch et al. [2004].

[31] In this study, the BUM spectra have been used to estimate the generation altitude of the SEE. The electron gyroharmonic frequency changes according to the geomagnetic field strength with height. In addition, because of the changes in the interaction altitude of the pump wave due to pump-induced electron density variations [Ashrafi et al., 2006], the BUM emission generation altitude varies accordingly. Therefore by calculating the electron cyclotron frequency from the International Geomagnetic Reference Field (IGRF) (1945-2010) magnetic field model, the generation altitude for the BUM emission has been estimated using the following equation: $f_{\mathrm{BUM}}=2 f_{0}-n f_{\mathrm{ce}}$. The electron cyclotron frequency is given by $f_{\mathrm{ce}}=e B /(2 \pi m)$. In other words, separation of the peak of the BUM spectra from the Heater frequency is an indication of how far the Heater frequency is from the electron gyrofrequency at each altitude. Figure 2 shows the SEE spectra during a pumping cycle starting at 16:17 UT with a 14-s integration. SEE spectra from six sequential frequency sweeps of the spectrum analyzer are shown. A power offset of $10 \mathrm{~dB}$ has been added to each spectrum for presentation purposes. The broken lines indicate the filtered BUM spectra which have been used to estimate 


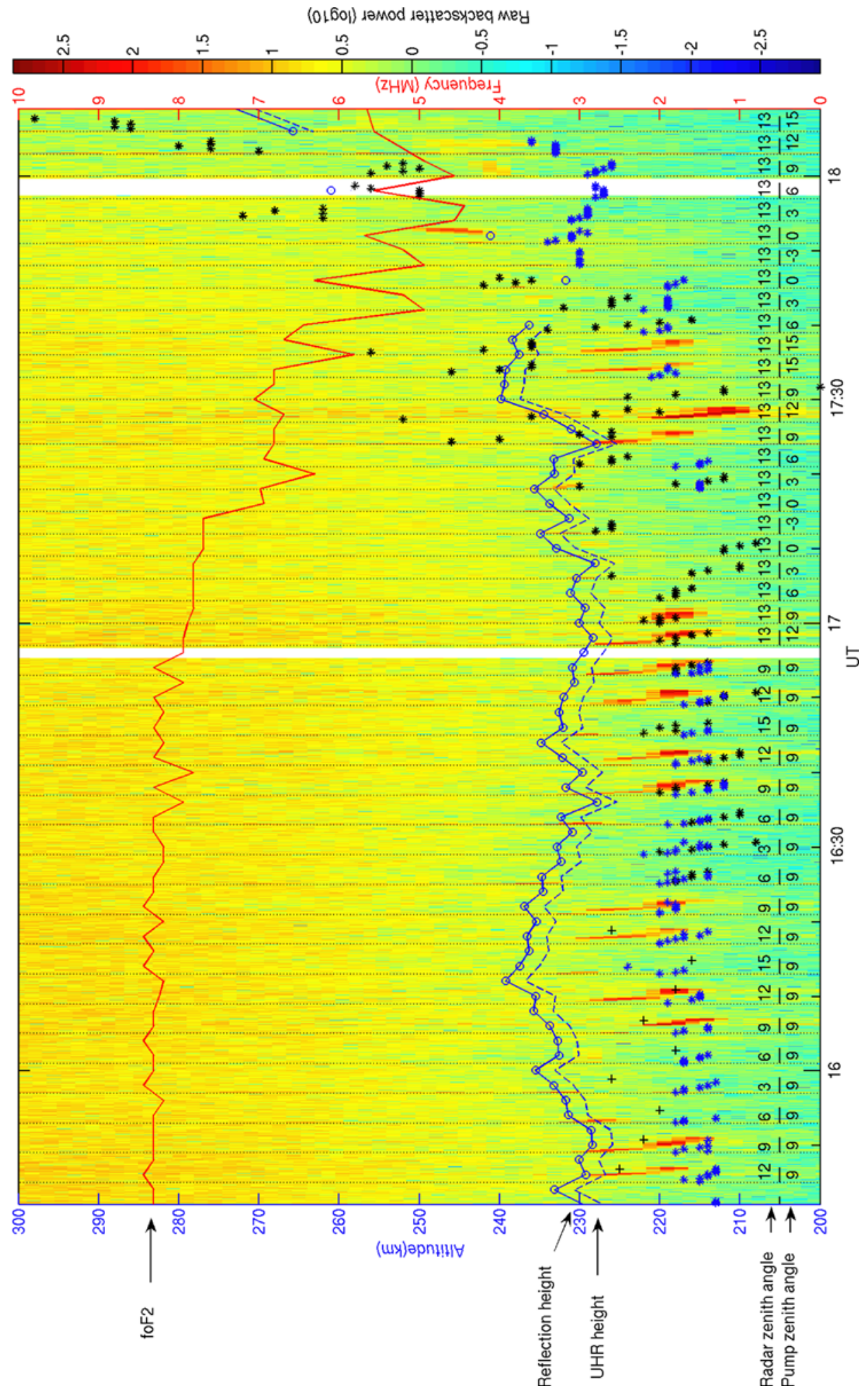

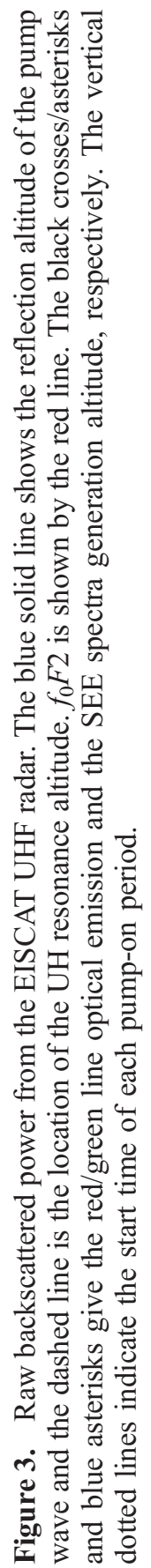


the peak of the spectra after median and low-pass filtering. The SEE spectra are filtered using a three-point running mean, spanning $900 \mathrm{~Hz}$, followed by a Savitsky-Golay smoothing filter. The heavy solid line joins the peak of the BUM spectra during the pumping cycle. The BUM peak frequency approaches the pump frequency which indicates descent of the SEE generation altitude.

[32] Combining all the processed observations, Figure 3 shows the optical height triangulation results (black crosses/ asterisks) and height estimate of the SEE generation region (blue asterisks) plotted on top of the EISCAT UHF radar backscatter power for more than 40 consecutive HF pump cycles. The intervals indicated by black lines show the pump-on periods. The upper labels mark the radar zenith angles south of vertical. The lower labels mark the pump beam zenith angles south of vertical (negative angles indicate north of vertical). The reflection height of the pump wave has been calculated using the ionosonde (coherent HF vertical backscatter radar) data. The UH resonance altitude, indicated by the dashed line, has been produced according to $f_{\mathrm{uh}}^{2}=f_{\mathrm{pe}}^{2}+f_{\mathrm{ce}}^{2}$ [e.g., Dysthe et al., 1982] where $f_{\mathrm{uh}}$ is the $\mathrm{UH}$ resonance frequency and $f_{\mathrm{pe}}$ is the local cold plasma frequency. For some of the pump cycles, there is no clear and consistent BUM structure throughout the pumping period, which is the reason why some of the SEE altitude estimates are missing.

[33] The height of the radar ion line enhancements indicates the region where the electrostatic Langmuir waves are excited as the result of plasma turbulence processes. The height determined from the SEE spectra occurs where the excited electrostatic waves scatter off the existing plasma irregularities and stimulate electromagnetic waves in the sidebands of the pump wave, which then can be detected on the ground. The optical emission height is the altitude at which accelerated electrons excite the ambient neutrals and produce the photons.

[34] The change in height of the brightest part of the optical emission has been calculated for the time period between 15:42 and 18:09 UT, as described earlier. Height estimates of the quasi-steady-state red line emission are shown for the first part of the experiment (15:42-16:21 UT) with black crosses. The second part of the experiment (16:25-18:09 UT) shows the estimated altitudes for the green line emission data (black asterisks). The quasi-steadystate location of the red line emission is consistently higher than the green line estimated altitude. This is most likely due to the long lifetime of the red line emission and descent of the interaction region, which never reaches a steady state for a full $30 \mathrm{~s}$; that is, the effective height of the emission is the true height of the steady state blob but from $\sim 30$ s prior to the measurements.

[35] The descent in altitude of the ion line data, shown in Figure 3, is a result of the descent of the pump waves' resonance region where the plasma frequency equals the pump frequency. The pump wave resonance altitude is simply related to the matching height [Stubbe et al., 1992]. Pumping the lower F-layer plasma causes an increase in electron temperature [Stocker et al., 1992; Rietveld et al., 2003] and decrease in electron recombination rate [Gurevich, 1978], thereby increasing the plasma density. Electron density increases near the reflection point of the radio waves, resulting in the descent of the reflection level of the pump wave [Ashrafi et al., 2006]. The general descent in height of the optical signature is consistent with the height variations of the EISCAT backscatter enhancements and changes in the SEE generation altitude.

[36] The SEE generation altitude is always below the UH altitude and below the ion line enhancements height. The altitude of the optical emission is mostly above the SEE generation height throughout the experiment. For the first half of the experiment (until 17:24 UT), the optical emissions appear below the reflection height and UH resonance altitude. After $\sim 17: 24 \mathrm{UT}$, with decreasing $f_{0} F 2$, the height of the optical signature starts to increase and appears to be sometimes above the reflection altitude. After $\sim 17: 40 \mathrm{UT}$, the weakening of the ionosphere leads to the ascent of the reflection altitude of the pump waves. In this period, the altitude of SEE formation increases and the optical emission production height also increases. Although this effect can be due to an increase in altitude of the electron acceleration region, the increasing vertical extent of the optical structure above the acceleration region also causes the estimated height of the optical emission column to increase.

[37] CUTLASS clearly shows weak enhancements $(\sim 20 \mathrm{~dB})$ in the backscatter power over the EISCAT HF beam (not shown), which is evidence of the existence of FAS in the vicinity of the UH resonance altitude. Typical backscatter power due to O-mode pumping can be $30 \mathrm{~dB}$ or more. In this case, although CUTLASS only makes a single observation $70 \mathrm{~s}$ into each pump cycle, the FAS will be fully developed at that time.

\section{Discussion}

[38] The aim of this study is to understand the electron acceleration mechanism involved in the production of the artificial optical emissions. Normally, when pumping near the electron gyrofrequency, the optical emissions are suppressed [Leyser et al., 2000; Kosch et al., 2002]. However, the November 2001 experiment is an exception, as the enhancements in the optical emission and the novel annuli structure were observed for the first time by pumping close to and slightly above the fourth electron gyroharmonic frequency. Other recent experiments at the HAARP facility in Alaska have also provided evidence that the optical emission maximizes at frequencies just above the electron gyroharmonics [Kosch et al., 2005; Gustavsson et al., 2006], which has been predicted in theory [Grach, 1979; Das and Fejer, 1979]. It is believed that both $\mathrm{PDI}_{\mathrm{UH} / \mathrm{EB}^{-}}$and TPI-related processes coexist and contribute to electron acceleration at frequencies close to and slightly above the gyroharmonic [Mishin et al., 2005b].

[39] The incoherent scatter ion line enhancements persist throughout the pump cycle only in the magnetic field-aligned and $9^{\circ} \mathrm{S}$ radar positions. However, short-lived ion line enhancements $(\sim 10-15 \mathrm{~s})$ have been detected at $6^{\circ}$ and $15^{\circ} \mathrm{S}$ radar zenith angles. These are the data that have been interpreted as evidence of a ring structure, detected by the radar during the first minute of pumping [Kosch et al., 2004]. Similar short-lived effects are detected after 16:57 UT with the radar pointing along the field line and the HF beam scanning from $3^{\circ}$ north to $15^{\circ}$ south of the zenith in $3^{\circ}$ steps. Strong backscatter enhancements are visible for $\mathrm{HF}$ beam zenith angles of $9^{\circ}$ and $12^{\circ} \mathrm{S}$ of vertical. 
[40] At gyroharmonic frequencies, HF radar backscatter power [Honary et al., 1999], anomalous absorption [Stocker et al., 1993; Stubbe et al., 1994], electron temperature enhancements [Honary et al., 1995; Robinson et al., 1996], and optical emissions [Kosch et al., 2002] minimize. The phenomena are all related to the suppression of FAS at frequencies close to the third or higher electron gyroharmonics. This suppression results in the reduced ability of FAS to trap the UH electrostatic waves near the electron gyrofrequencies [Mjølhus, 1993]. Therefore it is consistent with our special case where enhancements in the artificial optical emission with the presence of persistent ion line enhancements suggest that Langmuir turbulence-related processes are the main acceleration mechanism for the electrons [Kosch et al., 2004]. However, existence of the weak FAS in the CUTLASS backscatter is an indication that the UH waves are also present throughout the pumping period, in agreement with the mechanism described by Mishin et al. [2005a], which allows for the scattering of pump waves into Langmuir waves in the presence of $\mathrm{UH}$ waves, or equivalently FAS, for zenith angles greater than the Spitze angle $\left(\sim 6^{\circ}\right.$ at EISCAT). The ion line enhancements observed in the EISCAT UHF radar are most probably due to this mechanism, as they are not observed at zenith angles smaller (closer to vertical) than the Spitze angle.

[41] The BUM $-3 \mathrm{~dB}$ spectral width can be used to calculate the altitude range where the integrated SEE emissions come from using the following equation: $f_{\mathrm{C}}=$ $-f_{\mathrm{BUM}} / n$, where $n$ is the number of the harmonic [Leyser et al., 1990]. In our case, the spectral width of the BUM at $-3 \mathrm{~dB}$ is $\sim 15-30 \mathrm{kHz}$ which corresponds to a $3.75-7.5 \mathrm{kHz}$ change in the electron cyclotron frequency and is equivalent to a $\sim 8-12 \mathrm{~km}$ altitude range near the $\mathrm{UH}$ resonance height $(\sim 220-240 \mathrm{~km})$. Later in the experiment, after 17:30 UT when the reflection height and the SEE generation altitude are ascending as the ionosphere decays, the spectral width of the BUM increases to $35-45 \mathrm{kHz}$; hence, the vertical extent of the BUM generation region also increases to $\sim 15-20 \mathrm{~km}$. The estimated vertical extent is consistent with the BUM features being linked to the existence of FAS in the vicinity of the UH resonance altitude. The elongation of the FAS in the magnetic field line direction has been estimated to be $\sim 10$ $15 \mathrm{~km}$ [Jones et al., 1984; Kelley et al., 1995]. Using multifrequency HF radar measurements, e-folding scale lengths of $\sim 20 \mathrm{~km}$ have been measured for the density perturbations near the UH resonance altitude [Senior et al., 2004]. It is important to point out that the generation region of the SEE spectra is always below the UH altitude and below the ion line enhancements region. From Figure 3, it is clear that the BUM spectra emanate from the bottom part of the FAS below the UH resonance height. This phenomenon is probably due to the scattering of the SEE waves from the upper reaches of the FAS which prevent these SEE waves from reaching the ground; that is, only the SEE generation region from the bottom of the FAS is visible from the ground because the FAS themselves scatter EM waves; so any SEE generated above the UH resonance altitude is lost to anomalous absorption.

[42] It has been theorized that by pumping near the gyroresonances a small fraction of the energy of the pump wave changes the distribution function of the accelerated electrons and causes the distribution to have a rather long tail through to high energies up to approximately $1 \mathrm{keV}$ [Vas 'kov and Ivanov-Kholodniy, 1991]. Another theoretical study has also shown formation of an intense electron distribution function tail at high energies as a result of the modifying waves with frequencies close to harmonics of the electron cyclotron frequency [Dimant et al., 1992]. The high pump ERP used, combined with pumping just above the fourth gyroharmonic, most likely explains the unusually high optical intensities during the experiment, up to $300 \mathrm{R}$ for red line and $100 \mathrm{R}$ for green line emissions.

\section{Conclusion}

[43] The experimental results of ionospheric pumping at a frequency close to and above the fourth electron gyrofrequency at Tromsø have been presented. Several phenomena were observed:

[44] (1) Artificial optical annuli appeared for pumping at $9^{\circ} \mathrm{S}$ of local geographic zenith. The collapse of the ring into a blob and descent in altitude onto the magnetic field line direction was observed.

[45] (2) Persistent descending ion line enhancements, consistent with the optical data, were observed only in directions close to the magnetic field line $\left(9^{\circ}\right.$ and $12^{\circ} \mathrm{S}$ of local geographic zenith).

[46] (3) Short-lived $(\sim 5-10$ s) ion line enhancements were only observed at $6^{\circ}$ and $15^{\circ}$ south of vertical.

[47] (4) The BUM feature was observed in the SEE spectra throughout the experiment and changes in a way consistent with the optical and radar signatures' descent in altitude.

[48] The altitudes of the artificial optical emission, the SEE generation region, and the incoherent backscatter enhancements in the radar data have been compared. Significant results of this study include the following:

[49] (1) For a steady state ionosphere, the spatial evolution of the optical signature is consistent with the descent of the SEE BUM spectra and ion line enhancements in the radar backscattered power. The altitude of the optical emission is mostly above the SEE generation altitude.

[50] (2) The artificial optical aurora is extended vertically along the magnetic field line above and below its maximum intensity region for a few tens of kilometers.

[51] (3) The observed SEE BUM generation altitude is always below that of the ion line enhancements and extends in altitude for less than $\sim 20 \mathrm{~km}$, which is consistent with the elongation of FAS.

[52] Several different wave-plasma interaction processes resulting in electron acceleration are possible. Our observations show the following:

[53] (1) The CUTLASS backscatter enhancements are evidence of the existence of FAS and UH waves throughout the experiment, consistent with the generation of the SEE BUM spectra, which result from the TPI mechanism.

[54] (2) The EISCAT UHF backscatter enhancements are evidence of the existence of Langmuir turbulence.

[55] (3) Both the Langmuir waves and UH resonance are likely to contribute simultaneously to the acceleration of electrons to produce the high-intensity optical emissions observed. 
[56] Many aspects of this experiment are not fully understood and require further study.

[57] Acknowledgments. DASI was operated by the Max Planck Institute for Aeronomy (Katlenburg-Lindau, Germany) in collaboration with the Auroral Observatory (Tromsø, Norway). EISCAT is an international scientific association supported by the research councils of Finland, France, Germany, Japan, Norway, Sweden and the United Kingdom.

[58] Amitava Bhattacharjee thanks Evgeny Mishin and another reviewer for their assistance in evaluating this paper.

\section{References}

Armstrong, W. T., R. Massey, P. Argo, R. Carlos, D. Riggin, P. Y. Cheung, M. McCarrick, J. Stanley, and A. Y. Wong (1990), Continuous measurement of stimulated electromagnetic emission spectra from HF excited ionospheric turbulence, Radio Sci., 25, 1283-1289.

Ashrafi, M., M. J. Kosch, and K. Kaila (2005), Height triangulation of artificial optical emissions in the F-layer, paper presented at 31 Annual European Meeting on Atmospheric Studies by Optical Methods, Ambleside, UK, August 2004.

Ashrafi, M., M. J. Kosch, and F. Honary (2006), Heater-induced altitude descent of the EISCAT UHF ion line enhancements: Observations and modelling, Adv. Space Res., 38, 2645-2652.

Bernhardt, P. A., C. A. Tepley, and L. M. Duncan (1989), Airglow enhancements associated with plasma cavities formed during ionospheric heating experiments, J. Geophys. Res., 94, 9071-9092.

Bernhardt, P. A., W. A. Scales, S. M. Grach, A. N. Keroshtin, and D. S Kotik (1991), Excitation of artificial airglow by high power radio waves from the "SURA" ionospheric heating facility, Geophys. Res. Lett., 18, $1477-1480$.

Birkmayer, W., T. Hagfors, and W. Kofman (1986), Small-scale plasmadensity depletions in Arecibo high-frequency modification experiments, Phys. Rev. Lett., 57, 1008-1011.

Cheung, P. Y., A. Y. Wong, J. Pau, and E. Mjølhus (1998), Controlled ionospheric preconditioning and stimulated electromagnetic radiation, Phys. Rev. Lett., 58, 4891-4894.

Das, A. C., and J. A. Fejer (1979), Resonance instability of small-scale field-aligned irregularities, J. Geophys. Res., 84, 6701-6704.

Dhillon, R. S., and T. R. Robinson (2005), Observations of time dependence and aspect sensitivity of regions of enhanced UHF backscatter associated with RF heating, Ann. Geophys., 23(1), 75-85.

Dimant, Y. S., A. V. Gurevich, and K. P. Zybin (1992), Acceleration of electrons in the ionosphere under the action of intense radio-waves near electron cyclotron harmonics, J. Atmos. Terr. Phys., 54, 425-436.

Djuth, F. T., P. Stubbe, M. P. Sulzer, H. Kohl, M. T. Rietveld, and J. H. Elder (1994), Altitude characteristics of plasma turbulence excited with the Tromsø superheater, J. Geophys. Res., 99(A1), 333-339.

Djuth, F. T., B. Isham, M. T. Rietveld, T. Hagfors, and C. La Hoz (2004), First $100 \mathrm{~ms}$ of HF modification at Tromsø, Norway, J. Geophys. Res., 109, A11307, doi:10.1029/2003JA010236.

DuBois, D. F., A. H. H. A. Rose, and D. Russell (1993), Space and time distribution of HF excited Langmuir turbulence in the ionosphere: Comparison of theory and experiment, J. Geophys. Res., 98, 17,543-17,567.

Duffet-Smith, P. (1990), Practical Astronomy with Your Calculator, Cambridge University Press, London.

Dysthe, K. B., E. Mjølhus, H. Pecscli, and K. Rypdal (1982), Thermal cavitons, Phys. Scr., T2/2, 548-559.

Dysthe, K. B., E. Mjølhus, H. Pecscli, and K. Rypdal (1983), A thermal oscillating two stream instability, J. Geophys. Res., 26, 146-157.

Fejer, J. A. (1979), Ionospheric modification and parametric instabilities, Rev. Geophys. Space Phys., 17, 135-153.

Frolov, V. L., S. M. Grach, L. M. Erukhimov, G. P. Komrakov, E. N. Sergeev, B. Thidé, and T. Carozzi (1996), Peculiarities in the evolution of the BUM of stimulated radio emission of the ionosphere, Radiophys Quantum Electron., 39, 352-371.

Frolov, V. L., L. M. Ekukhimov, L. M. Kagan, G. P. Komrakov, E. N Sergeev, and P. Stubbe (1998), Two-component nature of the broad up-shifted maximum in stimulated electromagnetic emission spectra, Phys. Rev. Lett., 81, 1630-1633

Grach, S. M. (1979), Thermal parametric instability in ionospheric plasma at frequencies close to $\omega_{\mathrm{He}}$ and $2 \omega_{\mathrm{He}}$, Radiophys. Quantum Electron., 42 , $572-588$

Grach, S. M. (1985), Electromagnetic radiation from artificial ionospheric plasma turbulence, Radiophys. Quantum Electron., 28, 470-477.

Grach, S. M., A. N. Karashtin, N. A. Mityakov, V. O. Rapoport, and V. Y. Trakhtengerts (1977), Parametric interaction between electromagnetic radiation and the ionospheric plasma, Radiophys. Quantum Electron., $20,1254-1258$
Grach, S., E. Sergeev, A. Nasyrov, R. Gumerov, R. Shaimukhametov, I. Nasyrov, and G. Komrakov (2004), Simultaneous observations of the $557.7 \mathrm{~nm}$ airglow and stimulated electromagnetic emission during $\mathrm{HF}$ pumping of the ionosphere with diagnostic schedule: First results, $A d v$. Space Res., 34(11), 2241-2466.

Gumerov, R. I., V. B. Kapkov, G. P. Komrakov, and A. M. Nasyrov (1999), Artificial ionospheric glow caused by the short-term effect of high-power RF radiation, Radiophys. Quantum Electron., 42, 524-527.

Gurevich, A. V. (1978), Nonlinear Phenomena in the Ionosphere, Springer, New York.

Gurevich, A. V., Y. A. Dimant, G. M. Milikh, and V. V. Vas'kov (1985), Multiple acceleration of electrons in the regions of high-power radiowave reflecton in the ionosphere, J. Atmos. Terr. Phys., 47, 1057-1070.

Gustavsson, B., et al. (2001), First tomographic estimate of volume distribution of HF-pump enhanced airglow emission, J. Geophys. Res., 106, $29,105-29,124$

Gustavsson, B., B. U. E. Brändström, Å. Steen, T. Sergienko, T. B. Leyser, M. T. Rietveld, T. Aso, and M. Ejiri (2002), Nearly simultaneous images of HF-pump enhanced airglow at $6300 \mathrm{~A}$ and $5577 \AA$, Geophys. Res. Lett., 29(24), 2220, doi:10.1029/2002GL015350.

Gustavsson, B., T. Sergienko, I. Häggström, and F. Honary (2004), Simulation of high energy tail of electron distribution function, $A d v$. Polar $U p$. Atmos. Res., 18, 1-9.

Gustavsson, B., T. B. Leyser, M. Kosch, M. T. Rietveld, A. Steen, B. U. E. Brändström, and T. Aso (2006), Electron gyroharmonic effects in ionization and electron acceleration during hf pumping in the ionosphere, Phys. Rev. Lett., in press.

Guzdar, P. N., P. K. Chaturvedi, K. Papadopoulos, M. J. Keskinen, and S. L. Ossakow (1996), The self-focusing instability in the presence of density irregularities in the ionosphere, J. Geophys. Res., 101, 24532460

Haslett, J. C., and L. R. Megill (1974), A model of enhanced airglow excited RF-radiation, Radio Sci., 9, 1005-1019.

Honary, F., A. J. Stocker, T. R. Robinson, T. B. Jones, and P. Stubbe (1995), Ionospheric plasma response to $\mathrm{HF}$ radio waves operating at frequencies close to the third harmonic of the electron gyrofrequency, J. Geophys. Res., 101, 21,489-21,502.

Honary, F., T. R. Robinson, D. M. Wright, A. J. Stocker, M. T. Rietveld, and I. McCrea (1999), First direct observations of the reduced striations at pump frequencies close to the electron gyroharmonics, Ann. Geophys., 17(A4), 1235-1238.

Isham, B., C. L. Hoz, M. T. Rietveld, T. Hagfors, and T. B. Leyser (1999a), Cavitating Langmuir turbulence observed during EISCAT high latitude ionospheric interaction experiments, Phys. Rev. Lett., 83(13), 25762579 .

Isham, B., M. T. Rietveld, T. Hagfors, C. L. Hoz, E. Mishin, W. Kofman, T. B. Leyser, and A. P. van Eyken (1999b), Aspect angle dependence of HF enhanced incoherent backscatter, Adv. Space Res., 16, 1003-1006. stomin, Y. N., and T. B. Leyser (1995), Parametric decay of an electromagnetic wave near electron cyclotron harmonics, Phys. Plasmas, 2(6), 2084-2097.

Istomin, Y. N., and T. B. Leyser (2003), Electron acceleration by cylindrical upper hybrid oscillations trapped in density irregularities in the ionosphere, Phys. Plasmas, 10, 2962-2970.

Jones, T. B., T. R. Robinson, P. Stubbe, and H. Kopka (1984), Frequency dependence of anomalous absorption caused by high power radio waves, J. Atmos. Terr. Phys., 46, 147-153.

Jones, T. B., T. Robinson, P. Stubbe, and H. Kopka (1986), EISCAT observations of heated ionosphere, J. Atmos. Terr. Phys., 48(A1), $1027-1035$

Kelley, M. C., T. L. Arce, J. Salowey, S. W. T. M. Armstrong, M. Carter, and L. Duncan (1995), Density depletions at the $10 \mathrm{~m}$ scale induced by the Arecibo heater, J. Geophys. Res., 100, 17,367-17,376.

Kohl, H., H. Kopka, P. Stubbe, and M. Rietveld (1993), Introduction to ionospheric heating experiments at Tromsø: Part 2. Scientific problems, J. Atmos. Terr. Phys. 55(4/5), 601-613.

Kosch, M. J., T. Hagfors, and E. Nielsen (1998), A new digital all-sky imager for optical auroral studies in conjunction with the Scandinavian twin auroral radar experiment, Rev. Sci. Instrum., 69, 578-584.

Kosch, M. J., M. T. Rietveld, A. J. Kavanagh, C. Davis, T. K. Yeoman, F. Honary, and T. Hagfors (2002), High-latitude pump-induced optical emissions for frequencies close to the third electron gyro-harmonic, Geophys. Res. Lett., 29(3), 2112, doi:10.1029/2002GL015744.

Kosch, M. J., M. T. Rietveld, A. Senior, I. McCrea, A. J. Kavanagh, B. Isham, and F. Honary (2004), Novel artificial optical annular structures in the high latitude ionosphere over EISCAT, Geophys. Res. Lett., 31, L12805, doi:10.1029/2004GL019713.

Kosch, M. J., T. Pedersen, J. Hughes, R. Marshall, E. Gerken, A. Senior, D. Sentman, M. McCarrick, and F. T. Djuth (2005), Artificial optical 
emissions at HAARP for pump frequencies near the third and second electron gyro-harmonic, Ann. Geophys., 23, 1585-1592.

Kuo, S. P., M. C. Lee, and P. Kossey (1997), Excitation of oscillating two stream instability by upper hybrid pump waves in ionospheric heating experiments at Tromsø, Geophys. Res. Lett., 24(A10), 2969-2972.

Leyser, T. B. (2001), Stimulated electromagnetic emissions by highfrequency electromagnetic pumping of the ionospheric plasma, Space Sci. Rev., 98, 223-328.

Leyser, T. B., B. Thidé, H. Derblom, A. Hedberg, B. Lundborg, P. Stubbe, and H. Kopka (1989), Stimulated electromagnetic emission near electron cyclotron harmonics in the ionosphere, Phys. Rev. Lett., 63, 1145-1147.

Leyser, T., B. Thidé, H. Derblom, A. Hedberg, B. Lundborg, P. Stubbe, and H. Kopka (1990), Dependence of stimulated electromagnetic emission on the ionosphere and pump wave, J. Geophys. Res., 95, 17,233-17,244.

Leyser, T. B., B. Gustavsson, B. U. E. Brändström, F. Honary, Å. Steen, T. Aso, M. T. Rietveld, and M. Ejiri (2000), Simultaneous measurements of high-frequency pump-enhanced airglow and ionospheric temperatures at auroral latitudes, Adv. Polar Up. Atmos. Res., 14, 1-11.

Mantas, G. P. (1994), Large 6300- airglow intensity enhancements observed in ionosphere heating experiments are excited by thermal electrons, J. Geophys. Res., 99, 8993-9002.

Mantas, G. P., and H. C. Carlson (1996), Reinterpretation of the 6300airglow enhancements observed in ionosphere heating experiments based on analysis of Platteville, Colorado, data, J. Geophys. Res., 101, 195209.

Mishin, E. V., W. J. Burke, and T. Pedersen (2004), On the onset of HF-induced airglow at HAARP, J. Geophys. Res., 109(6), A02305, doi:10.1029/2003JA010205.

Mishin, E. V., W. J. Burke, and T. Pedersen (2005a), HF-induced airglow at magnetic zenith: Theoretical considerations, Ann. Geophys., 23(1), $47-$ 53.

Mishin, E. V., M. J. Kosch, T. R. Pedersen, and W. J. Burke (2005b), HF-induced airglow at magnetic zenith: Thermal and parametric instabilities near electron gyroharmonics, Geophys. Res. Lett., 32, L23106, doi:10.1029/2005GL023864.

Mjølhus, E. (1993), On the small scale striation effect in ionospheric radio modification experiments near harmonics of the electron gyrofrequency, Radio Sci., 25, 1321-1339.

Pedersen, T. R., M. McCarrick, E. Gerken, C. Selcher, D. Sentman, H. Carlson, and A. Gurevich (2003), Magnetic zenith enhancement of HF radio-induced airglow production at HAARP, Geophys. Res. Lett., 30(4), 1169, doi:10.1029/2002GL026096.

Rietveld, M. T., H. Kohl, H. Kopka, and P. Stubbe (1993), Introduction to ionospheric heating at Tromsø: 1. Experimental overview, J. Atmos. Terr Phys., 55, 577-599.

Rietveld, M. T., B. Isham, H. Kohl, C. L. Hoz, and T. Hagfors (2000), Measurements of HF-enhanced plasma and ion lines at EISCAT with high altitude resolution, J. Geophys. Res., 105, 7429-7439.

Rietveld, M. T., M. J. Kosch, N. F. Blagoveshchenskaya, V. A. Kornienko, T. B. Leyser, and T. K. Yeoman (2003), Ionospheric electron heating, optical emissions, and striations induced by powerful HF radio waves at high latitudes: Aspect angle dependence, J. Geophys. Res., 108(A4), 1141, doi:10.1029/2002JA009543

Rishbeth, H., and A. P. van Eyken (1993), EISCAT: Early history and the first ten years of operation, J. Atmos. Terr. Phys., 55, 525-542.
Robinson, T. R. (1989), The heating of the high latitude ionosphere by high power radio waves, Phys. Rep., 179(2/3), 79-209.

Robinson, T. R., F. Honary, A. Stocker, T. Jones, and P. Stubbe (1996), First EISCAT observations of the modification of F-region electron temperature during RF heating at harmonics of the electron gyrofrequency, J. Atmos. Terr. Phys., 58, 385-395.

Senior, A., N. D. Borisov, M. J. Kosch, T. K. Yeoman, F. Honary, and M. T. Rietveld (2004), Multi-frequency HF radar measurements of artificial f-region field-aligned irregularities, Ann. Geophys., 22, 3503-3511.

Stocker, A. J., F. Honary, T. R. Robinson, T. B. Jones, P. Stubbe, and H. Kopka (1992), EISCAT observations of large scale electron temperature and electron density perturbations caused by high power HF radio waves, J. Atmos. Terr. Phys., 54(11/12), 1555-1572.

Stocker, A. J., F. Honary, T. R. Robinson, T. B. Jones, and P. Stubbe (1993), Anomalous absorption during artificial modification at harmonics of the electron gyrofrequency, J. Geophys. Res., 98, 13627-13634.

Störmer, C. (1955), The Polar Aurora, Oxford University Press, London.

Stubbe, P. (1996), Review of ionospheric modification experiments at Tromsø, J. Atmos. Terr. Phys., 58, 349-368.

Stubbe, P., et al. (1982), Ionospheric modification experiment in northern Scandinavia, J. Atmos. Terr. Phys, 44, 1025-1041.

Stubbe, P., H. Kopka, B. Thidé, and H. Derblom (1984), Stimulated electromagnetic emission: A new technique to study the parametric decay instability in the ionosphere, J. Geophys. Res., 89(A9), 7523-7536.

Stubbe, P., H. Kohl, and M. T. Rietveld (1992), Langmuir turbulence and ionospheric modification, J. Geophys. Res., 97(A5), 6285-6297.

Stubbe, P., A. J. Stocker, F. Honary, T. R. Robinson, and T. B. Jones (1994), Stimulated electromagnetic emissions (SEE) and anomalous HF wave absorption near electron gyroharmonics, J. Geophys. Res., 99, 62336246.

Thidé, B., H. Kopka, and P. Stubbe (1982), Observations of stimulated scattering of a strong high-frequency radio wave in the ionosphere, Phys. Rev. Lett., 49, 1561-1564.

Thidé, B., H. Derblom, A. Hedberg, H. Kopka, and P. Stubbe (1983), Observations of stimulated electromagnetic emissions in ionospheric heating experiments, Radio Sci., 18, 851-859.

Vas'kov, V. V., and A. V. Gurevich (1976), Non-linear resonance instability of a plasma in the field of an ordinary electromagnetic wave, Sov. Phys. JETP, 42, 91-97.

Vas'kov, V. V., and G. S. Ivanov-Kholodniy (1991), Acceleration of ionospheric plasma electrons by a high-power radio wave under conditions of multiple electron gyroresonance, Geomagn. Aeron., 31, 839-844.

Wagner, L. S., P. A. Berhnardt, J. A. Goldstein, C. A. Selcher, V. L. Frolov, and E. N. Sergeev (1999), Effect of ionospheric self-conditioning and preconditioning on the broad upshifted maximum component of stimulated electromagnetic emission, J. Geophys. Res., 104, 2573-2590.

M. Ashrafi, School of Physics and Astronomy, University of Southampton Highfield, Southampton, S017 1BJ, UK. (m.ashrafi@phys.soton. ac.uk)

B. Isham, Department of Electrical Engineering, Interamerican University of Puerto Rico, Bayamón, PR 00957, USA.

K. Kaila, Department of Physical Sciences, University of Oulu, Oulu, FIN-90570, Finland.

M. J. Kosch, Department of Communications Systems, InfoLab21, Lancaster University, Lancanshire, LA1 4WA, UK. 